\title{
ON THE q-ANALOG OF KUMIMER'S THEOREM AND APPLICATIONS
}

\author{
GEORGE E. ANDREWS
}

1. Introduction. The $q$-analogs for Gauss's summation of ${ }_{2} F_{1}[a, b ; c ; 1]$ and Saalschutz's summation of ${ }_{3} F_{2}[a, b,-n ; c, a+b-c-n+1 ; 1]$ are well known, namely, E. Heine [8; p. 107, Equation (6)] showed that

$$
{ }_{2} \phi_{1}\left[\begin{array}{c}
a, b ; q, c / a b \\
c
\end{array}\right]=\frac{(c / a)_{\infty}(c / b)_{\infty}}{(c)_{\infty}(c / a b)_{\infty}}
$$

where

$$
{ }_{m} \phi_{n}\left[\begin{array}{c}
a_{1}, \cdots, a_{m} ; q, z \\
b_{1}, \cdots, b_{n}
\end{array}\right]=\sum_{j=0}^{\infty} \frac{\left(a_{1}\right)_{i} \cdots\left(a_{m}\right)_{j} z^{j}}{(q)_{j}\left(b_{1}\right)_{i} \cdots\left(b_{n}\right)_{j}},
$$

and $(a)_{n}=(a ; q)_{n}=(1-a)(1-a q) \cdots\left(1-a q^{n-1}\right),(a)_{\infty}=(a ; q)_{\infty}=\lim _{n \rightarrow \infty}(a)_{n}$. (See also [12; p. 97, Equation (3.3.2.2)].) F. H. Jackson [9; p. 145] showed that

$$
{ }_{3} \phi_{2}\left[\begin{array}{c}
a, b, q^{-n} ; q, q \\
c, a b q / c q^{n}
\end{array}\right]=\frac{(c / a)_{n}(c / b)_{n}}{(c)_{n}(c / a b)_{n}} .
$$

The $q$-analog of Dixon's summation of ${ }_{3} F_{2}[a, b, c ; 1+a-b, 1+a-c ; 1]$ was more difficult to find, and indeed only a partial analog is true; namely, W. N. Bailey [5] and F. H. Jackson [10; p. 167, Equation (2)] proved that if $a=q^{-2 n}$ where $n$ is a positive integer, then

$$
{ }_{3} \phi_{2}\left[\begin{array}{c}
a, b, c ; q, \frac{q^{2} a^{\frac{1}{2}}}{b c} \\
\frac{a q}{b}, \frac{a q}{c}
\end{array}\right]=\frac{(b / a)_{\infty}(c / a)_{\infty}\left(q a^{\frac{1}{2}}\right)_{\infty}\left(b c a^{-\frac{1}{2}}\right)_{\infty}}{\left(b a^{-\frac{1}{2}}\right)_{\infty}\left(c a^{-\frac{1}{2}}\right)_{\infty}\left(a^{-1} q a\right)_{\infty}\left(b c a^{-1}\right)_{\infty}} .
$$

There are three other well-known summations for the ${ }_{2} F_{1}$ series, namely, Kummer's theorem [12; p. 243, Equation (III. 5)]

$$
{ }_{2} F_{1}[a, b ; 1+a-b ;-1]=\frac{\Gamma(1+a-b) \Gamma\left(1+\frac{a}{2}\right)}{\Gamma\left(\frac{1}{2}+\frac{a}{2}\right) \Gamma\left(1+\frac{a}{2}-b\right)},
$$

Gauss's second theorem [12; p. 243, Equation III. 6)]

Received December 23, 1972. The author was partially supported by National Science Foundation Grant GP-23774. 


$$
{ }_{2} F_{1}\left[a, b ; \frac{1}{2}+\frac{a}{2}+\frac{b}{2} ; \frac{1}{2}\right]=\frac{\Gamma\left(\frac{1}{2}\right) \Gamma\left(\frac{1}{2}+\frac{a}{2}+\frac{b}{2}\right)}{\Gamma\left(\frac{1}{2}+\frac{a}{2}\right) \Gamma\left(\frac{1}{2}+\frac{b}{2}\right)},
$$

and Bailey's theorem [12; p. 243, Equation (III. 7)]

$$
{ }_{2} F_{1}\left[a, 1-a ; c ; \frac{1}{2}\right]=\frac{\Gamma\left(\frac{c}{2}\right) \Gamma\left(\frac{1}{2}+\frac{c}{2}\right)}{\Gamma\left(\frac{c}{2}+\frac{a}{2}\right) \Gamma\left(\frac{1}{2}+\frac{c}{2}-\frac{a}{2}\right)} .
$$

Of these three, only a $q$-analog of Kummer's theorem is known, namely, [6; p. 711] (see also [5; p. 173])

$$
{ }_{2} \phi_{1}\left[\begin{array}{c}
a, b ; q,-\frac{q}{b} \\
\frac{q a}{b}
\end{array}\right]=\frac{\left(a q ; q^{2}\right)_{\infty}(-q)_{\infty}\left(q^{2} a / b^{2} ; q^{2}\right)_{\infty}}{(q a / b)_{\infty}(-q / b)_{\infty}} .
$$

The only known proof of (1.7) consists of a specialization of parameters in Jackson's summation of the well-poised ${ }_{6} \phi_{5}$.

Our object here is to provide a very simple proof of (1.7) and to show that the following $q$-analogs of Gauss's second theorem and Bailey's theorem hold:

$$
\begin{aligned}
& \sum_{n=0}^{\infty} \frac{(a)_{n}(b)_{n} q^{\frac{1}{2} n(n+1)}}{(q)_{n}\left(q a b ; q^{2}\right)_{n}}=\frac{(-q)_{\infty}\left(a q ; q^{2}\right)_{\infty}\left(b q ; q^{2}\right)_{\infty}}{\left(q a b ; q^{2}\right)_{\infty}}, \\
& \sum_{n=0}^{\infty} \frac{(b)_{n}(q / b)_{n} c^{n} q^{\frac{1}{3 n(n-1)}}}{\left(q^{2} ; q^{2}\right)_{n}(c)_{n}}=\frac{\left(q c / b ; q^{2}\right)_{\infty}\left(b c ; q^{2}\right)_{\infty}}{(c)_{\infty}} .
\end{aligned}
$$

2. The q-analog of Kummer's theorem. Here we utilize the summation technique that was successfully employed in [1], [2], [3] and [4]. We shall need the elementary summation [12; p. 92, Equation (3.2.2.11)]

$$
\sum_{n=0}^{\infty} \frac{(A)_{n} T^{n}}{(q)_{n}} \equiv{ }_{1} \phi_{0}[A ; q, T]=(A T)_{\infty} /(T)_{\infty} .
$$

Therefore

$$
\begin{aligned}
{ }_{2} \phi_{1}\left[\begin{array}{c}
a, b ; q,-q / b] \\
q a / b
\end{array}\right] & =\sum_{n=0}^{\infty} \frac{(a)_{n}(b)_{n}(-q / b)^{n}}{(q)_{n}(a q / b)_{n}} \\
& =\frac{(a)_{\infty}}{(a q / b)_{\infty}} \sum_{n=0}^{\infty} \frac{(b)_{n}(-q / b)^{n}\left(a q^{n+1} / b\right)_{\infty}}{(q)_{n}\left(a q^{n}\right)_{\infty}} \\
& =\frac{(a)_{\infty}}{(a q / b)_{\infty}} \sum_{n=0}^{\infty} \frac{(b)_{n}(-q / b)^{n}}{(q)_{n}} \sum_{m=0}^{\infty} \frac{(q / b)_{m} a^{m} q^{n m}}{(q)_{m}}
\end{aligned}
$$




$$
\begin{aligned}
& =\frac{(a)_{\infty}}{(a q / b)_{\infty}} \sum_{m=0}^{\infty} \frac{(q / b)_{m} a^{m}\left(-q^{m+1}\right)_{\infty}}{(q)_{m}\left(-q^{m+1} / b\right)_{\infty}} \\
& =\frac{(a)_{\infty}(-q)_{\infty}}{(a q / b)_{\infty}(-q / b)_{\infty}} \sum_{m=0}^{\infty} \frac{\left(q^{2} / b^{2} ; q^{2}\right)_{m} a^{m}}{\left(q^{2} ; q^{2}\right)_{m}} \\
& =\frac{(a)_{\infty}(-q)_{\infty}\left(a q^{2} / b^{2} ; q^{2}\right)_{\infty}}{(a q / b)_{\infty}(-q / b)\left(a ; q^{2}\right)_{\infty}} \\
& =\frac{\left(a q ; q^{2}\right)_{\infty}(-q)_{\infty}\left(a q^{2} / b^{2} ; q^{2}\right)_{\infty}}{(a q / b)_{\infty}(-q / b)_{\infty}}
\end{aligned}
$$

and so (1.7) is established. Technically what we have done is to set $\alpha=b$, $\beta=a, \gamma=q a / b$, and $\tau=-q / b$ in [3; Equation (I 1)]; we then have observed that the resulting ${ }_{2} \phi_{1}$ with base $q$ reduces to a ${ }_{1} \phi_{0}$ with base $q^{2}$.

3. The q-analogs of Gauss's second theorem and Bailey's theorem. As is well-known [12; p. 32], both (1.5) and (1.6) may be deduced by the application of Kummer's theorem to the following identity:

$$
(1-z)^{-a}{ }_{2} F_{1}\left[\begin{array}{c}
a, b ;-z /(1-z) \\
c
\end{array}\right]={ }_{2} F_{1}\left[\begin{array}{c}
a, c-b ; z \\
c
\end{array}\right] .
$$

The following lemma is the $q$-analog of (3.1), and from it we may deduce (1.8) and (1.9) utilizing the $q$-analog of Kummer's theorem. Actually this lemma was given by F. H. Jackson [9; p. 145, Equation (4)]; his proof entails the development of a $q$-analog of Euler's transformation of power series. We include a short proof that shows this result to be a limiting case of an identity of N. Hall [7].

$$
\text { Lemma. } \quad \sum_{n=0}^{\infty} \frac{(\beta)_{n}(\alpha)_{n}(-1)^{n} q^{\left(\begin{array}{c}
n \\
2
\end{array}\right)(x \gamma / \beta)^{n}}}{(q)_{n}(\gamma)_{n}(x \alpha)_{n}}=\frac{(x)_{\infty}}{(x \alpha)_{\infty}} \sum_{n=0}^{\infty} \frac{(\gamma / \beta)_{n}(\alpha)_{n} x^{n}}{(q)_{n}(\gamma)_{n}} .
$$

Proof. N. Hall [7] (see also [11; p. 174, Equation (10.1)]) has proved the result

$$
\begin{aligned}
{ }_{3} \phi_{2}\left[\begin{array}{r}
a_{1}, a_{2}, a_{3} ; q, b_{1} b_{2} / a_{1} a_{2} a_{3} \\
b_{1}, b_{2}
\end{array}\right] \\
\quad=\frac{\left(b_{2} / a_{3}\right)_{\infty}\left(b_{1} b_{2} / a_{1} a_{2}\right)_{\infty}}{\left(b_{2}\right)_{\infty}\left(b_{1} b_{2} / a_{1} a_{2} a_{3}\right)_{\infty}}{ }_{3} \phi_{2}\left[\begin{array}{c}
b_{1} / a_{1}, b_{1} / a_{2}, a_{3} ; q, b_{2} / a_{3} \\
b_{1}, b_{1} b_{2} / a_{1} a_{2}
\end{array}\right] .
\end{aligned}
$$

Our lemma follows directly by the substitutions $a_{1}=\beta, a_{3}=\alpha, b_{1}=\gamma, b_{2}=x \alpha$ if we then let $a_{2} \rightarrow \infty$.

To obtain (1.8), we set $\alpha=a, \beta=b, \gamma=q^{\frac{1}{2}} a^{\frac{1}{2}} b^{\frac{1}{2}}$ and $x=-q^{\frac{1}{2}} b^{\frac{1}{2}} a^{-\frac{1}{2}}$ in the lemma. Hence 


$$
\begin{aligned}
\sum_{n=0}^{\infty} \frac{(a)_{n}(b)_{n} q^{\frac{3}{3} n(n+1)}}{(q)_{n}\left(q a b ; q^{2}\right)_{n}} & =\frac{\left(-q^{\frac{1}{2}} b^{\frac{1}{2}} a^{-\frac{1}{2}}\right)_{\infty}}{\left(-q^{\frac{1}{2}} b^{\frac{1}{2}} a^{\frac{1}{2}}\right)_{\infty}}{ }_{2} \phi_{1}\left[\begin{array}{c}
\left.a, q^{\frac{1}{2}} a^{\frac{1}{2}} b^{-\frac{1}{2}} ; q,-q^{\frac{1}{2}} b^{\frac{1}{2}} a^{-\frac{1}{2}}\right] \\
q^{\frac{1}{2}} a^{\frac{1}{2}} b^{\frac{1}{2}}
\end{array}\right] \\
& =\frac{\left(-q^{\frac{1}{2}} b^{\frac{1}{2}} a^{-\frac{1}{2}}\right)_{\infty}}{\left(-q^{\frac{1}{2}} b^{\frac{1}{2}} a^{\frac{1}{2}}\right)_{\infty}} \frac{\left(a q ; q^{2}\right)_{\infty}(-q)_{\infty}\left(b q ; q^{2}\right)_{\infty}}{\left(q^{\frac{1}{2}} b^{\frac{1}{2}} a^{\frac{1}{2}}\right)_{\infty}\left(-q^{\frac{1}{3}} b^{\frac{1}{2}} a^{-\frac{1}{2}}\right)_{\infty}} \quad(\text { by }(1.7)) \\
& =\frac{(-q)_{\infty}\left(a q ; q^{2}\right)_{\infty}\left(b q ; q^{2}\right)_{\infty}}{\left(a b q ; q^{2}\right)_{\infty}}
\end{aligned}
$$

which is (1.8).

To obtain (1.9), we set $\alpha=q / b, \beta=b, \gamma=c$ and $x=-b$ in the lemma. Hence

$$
\begin{aligned}
& \sum_{n=0}^{\infty} \frac{(b)_{n}(q / b)_{n} c^{n} q^{\frac{1}{2} n(n-1)}}{(q)_{n}(c)_{n}(-q)_{n}}=\sum_{n=0}^{\infty} \frac{(b)_{n}(q / b)_{n} c^{n} q^{\frac{1}{2} n(n-1)}}{\left(q^{2} ; q^{2}\right)_{n}(c)_{n}} \\
& =\frac{(-b)_{\infty}}{(-q)_{\infty}}{ }_{2} \phi_{1}\left[\begin{array}{c}
c / b, q / b ; q,-b \\
c
\end{array}\right] \\
& =\frac{(-b)_{\infty}\left(q c / b ; q^{2}\right)_{\infty}(-q)_{\infty}\left(b c ; q^{2}\right)_{\infty}}{(-q)_{\infty}(c)_{\infty}(-b)_{\infty}} \\
& =\frac{\left(q c / b ; q^{2}\right)_{\infty}\left(b c ; q^{2}\right)_{\infty}}{(c)_{\infty}} \text {. }
\end{aligned}
$$

1. George E. Andrews, On basic hypergeometric series, mock theta functions, and partitions, I, Quart. J. Math., vol. 17(1966), pp. 64-80.

2. - On basic hypergeometric series, mock theta functions, and partitions, II, Quart. J. Math., vol. 17(1966), pp. 132-143.

3. - - -identities of Auluck, Carlitz, and Rogers, Duke Math. J., vol. 33(1966), pp. 575-581.

4. - Summations and transformations for basic Appell series, J. London Math. Soc., vol. 4(1972), pp. 618-622.

5. W. N. BAILeY, A note on certain q-identities, Quart. J. Math., vol. 12(1941), pp. 173-175.

6. J. A. Daum, The basic analogue of Kummer's theorem, Bull. Amer. Math. Soc., vol. 48(1942), pp. 711-713.

7. Newman A. HaLl, An algebraic identity, J. London Math. Soc., vol. 11(1936), p. 276.

8. Eduard Heine, Theorie der Kugelfunctionen, Berlin, Reimer, 1878.

9. F. H. JAckson, Transformations of $q$-series, Mess. Math., vol. 39(1910), pp. 145-153.

10. —, Certain q-identities, Quart. J. Math., vol. 12(1941), pp. 167-172.

11. D. B. Sears, On the transformation theory of basic hypergeometric functions, Proc. London Math. Soc., vol. 53(1951), pp. 158-180,

12. Lucy J. Slater, Generalized Hypergeometric Functions, Cambridge, Cambridge Univ. Press, 1966.

Department of Mathematics, Pennsylvania State University, University Park, Pennsyluania 16802 